\title{
Cortical Stress Reaction in Two Patients on Bisphosphonate Therapy: Case Report
}

\author{
Papatya TOGAY, Berna ÇELİK, Derya SOY BUĞDAYCI, Nurdan PAKER \\ Department of Physical Medicine and Rehabilitation, İstanbul Physical Medicine Rehabilitation \\ Training and Research Hospital, Istanbul, Turkey
}

\begin{abstract}
Bisphosphonates are widely used, effective medications of documented osteoporosis. They accumulate in bone and are released for months or years despite the termination of treatment. Cortical stress reaction and related low energy atypical fractures of femoral shaft are amongst the main complications in patients with osteoporosis who are on long-term bisphosphonate therapy. Contralateral femoral cortical stress reaction may also be observed. In this article, we present two female patients who admitted to our outpatient clinic with anterior thigh pain, and review their clinical presentation. Lateral cortical thickening of both femurs were diagnosed in both patients.

Keywords: Bisphosphonate; cortical stress reaction; osteoporosis.
\end{abstract}

Bisphosphonates are widely used, effective medications of documented osteoporosis. They accumulate in bone and are released for months or years despite the termination of treatment. Low energy atypical fractures of femoral shaft and pelvis in patients who are on long-term bisphosphonate therapy were described in 2005. Since then, many cases have been reported and it is speculated that accumulation of microdamage induced by severely suppressed bone turnover causes clinical fatigue fractures. ${ }^{1}$ These fractures are often preceded by pain in thigh and show lateral cortical thickening of femur that leads to an atypical fracture pattern radiologically. Contralateral femoral cortical stress reaction may also be observed. Here, we present two female patients with lateral cortical thickening of both femurs who received oral bisphosphonate treatment.

\section{CASE REPORT}

Case 1- A 73-year-old female patient presented with low back, knee, and anterior thigh pain. Pain severity was 8 over 10 point according to visual analog scale. She had difficulty in walking because of pain. She had a history of arthroscopic knee surgery for meniscal pathology in her left knee five years ago. She had chronic low back pain due to L4-5 disc herniation and lumbar spinal stenosis. She had been treated for osteoporosis with alendronate for two years, then with salmon calcitonin for two years and ibandronic acid for a year. Neurological examination was normal. Plain radiographs revealed bilateral lateral cortical thickening in the femur (Figure 1). Magnetic resonance imaging was done to rule out fracture. Bone scintigraphy showed increased uptake in bilateral femoral diaphysis. Routine biochemical 
tests were normal except increased urinary deoxypyridinoline. Urinary deoxypyridinoline level was $63 \mathrm{nM} / \mathrm{mMCre}$ (normal range: 3-7.4 nM/mMCre).

Bone mineral densities and T scores of L2-L4 vertebra and femur neck were $1.088 \mathrm{~g} / \mathrm{cm}^{2}$, -0.9 and $0.761 \mathrm{~g} / \mathrm{cm}^{2},-2$, respectively. After the radiographical evaluation, bisphosphonate treatment was stopped and bed rest was advised. Walking with crutches and activity restriction for two months were recommended. Analgesics, calcium and vitamin $\mathrm{D}$, and strontium ranelate were prescribed. Physiotherapy including strengthening exercises was planned. The patient was informed about the risk of fracture. It has been two years since the diagnosis. Pain severity decreased and there is no reported fracture.

Case 2-A 61-year-old female patient presented with anterior thigh pain and difficulty in walking. Pain severity was 7 over 10 point according to visual analog scale. She had been treated with alendronate $70 \mathrm{mg}$ per week with calcium plus vitamin $\mathrm{D}$ for two years. Plain radiographs of femur were taken upon clinical presentation. Cortical stress reaction was detected in both femoral shafts on plain radiographs (Figure 2).

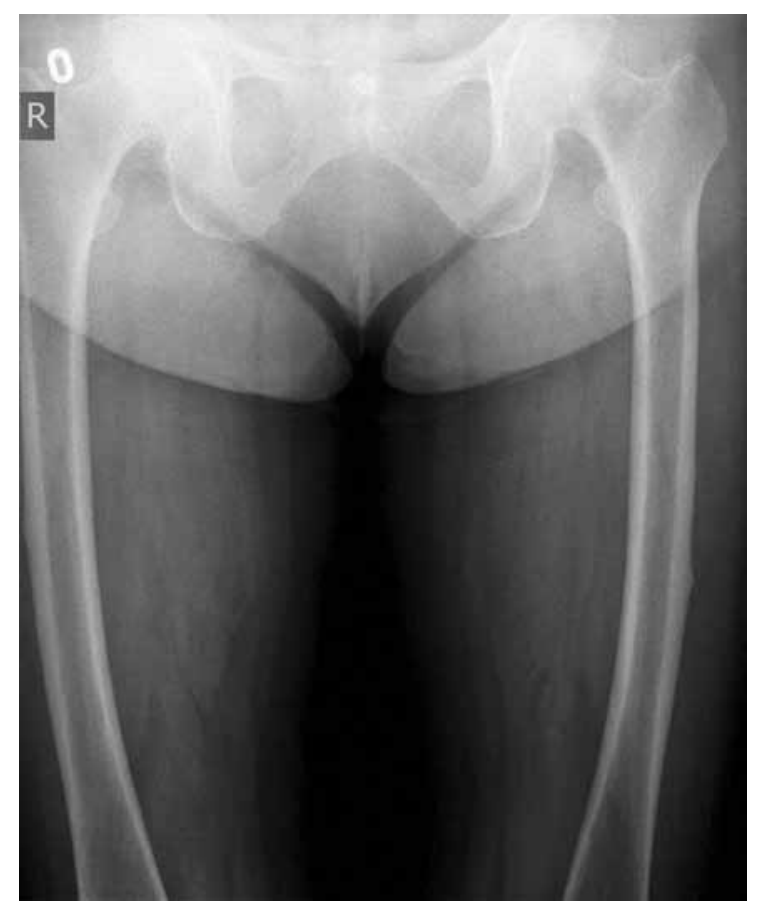

Figure 1. Plain radiography of the first patient (anteroposterior view).
Computed tomographic imaging was performed to rule out fracture (Figure 3). Bone scintigraphy showed increased uptake in distal $1 / 3$ ends of femoral shafts (Figure 4). Serum calcium value was normal and urinary deoxypiridinoline level was mildly increased (46 nM/mMCre).

At the time of diagnosis, dual-energy X-ray absorptiometry results were as follows: femur neck total bone mineral density was $0.782 \mathrm{~g} / \mathrm{cm}^{2}$ and $\mathrm{T}$ score was -1.8; L2-L4 bone mineral density was $0.865 \mathrm{~g} / \mathrm{cm}^{2}$ and $\mathrm{T}$ score was -2.8 .

After the diagnosis, we informed the patient about the cortical stress reaction. We advised her to restrict activities for two months and to use crutches during walking. Calcium and vitamin D supplements were prescribed along with strontium ranelate therapy. It has been one year since the diagnosis and her pain severity lessened and no fracture has been reported.

\section{DISCUSSION}

Bisphosphonates are prescribed as the first choice treatment in patients with osteoporosis and they strongly inhibit bone resorption. ${ }^{2}$ They are safe and

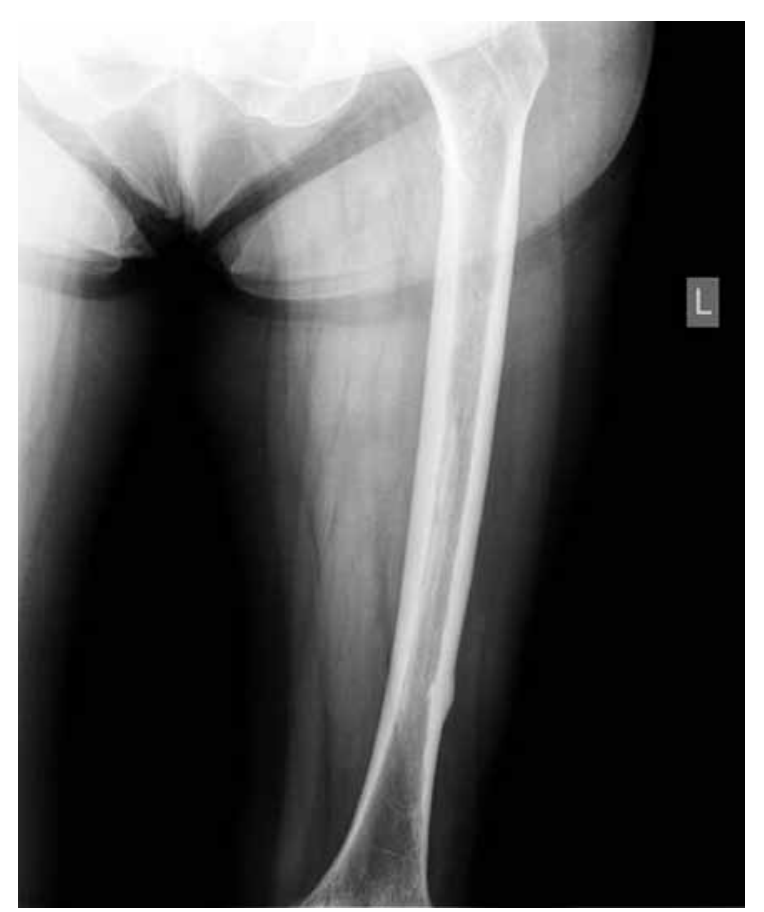

Figure 2. Plain radiography of the second patient (anteroposterior view). 


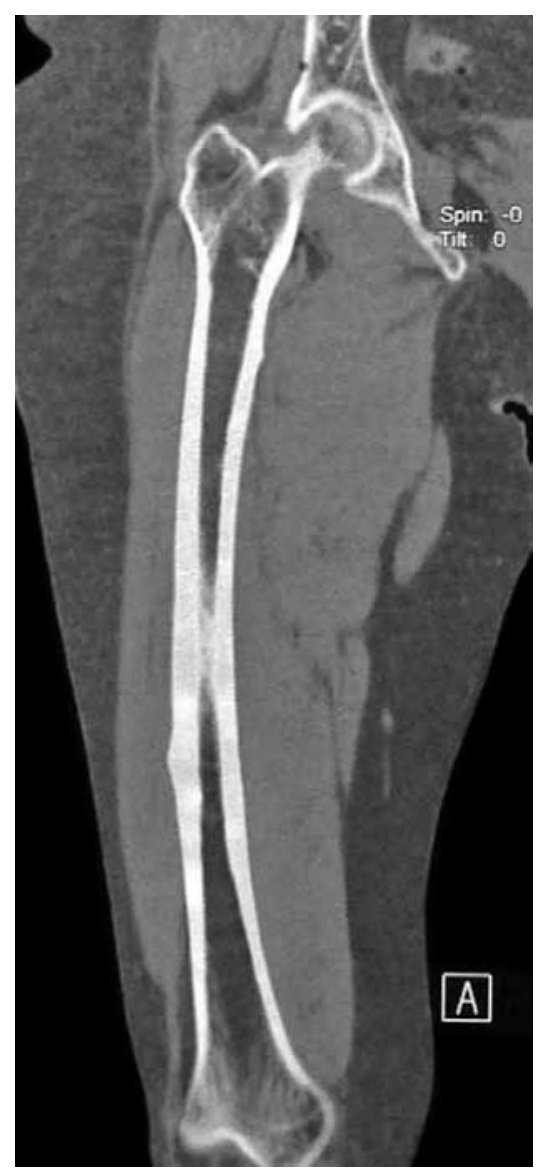

Figure 3. Computed tomographic imaging of the second patient.

effective when the duration of therapy is less than five years. Prolonged bisphosphonate therapy may contribute to atypical subtrochanteric fractures by over suppressing bone remodeling leading to alterations in bone mineral and organic matrix. ${ }^{3}$ As a consequence of the reduction in bone turnover by bisphosphonates, the mineralization of bone is significantly altered. Bisphosphonates prolong the life of existing remodeling units and reduce the formation of new ones. This prolongation causes most of the bone remodeling units to become older and fully mineralized, leading to an increased homogeneity of mineralization. ${ }^{3}$ Although increased mineralization is positively correlated with increased stiffness, it is negatively correlated with endurance. Reductions in toughness are closely predicted by changes in mineralization and would contribute to increased brittleness of the bone and susceptibility to fracture. ${ }^{3}$ Common features of these atypical

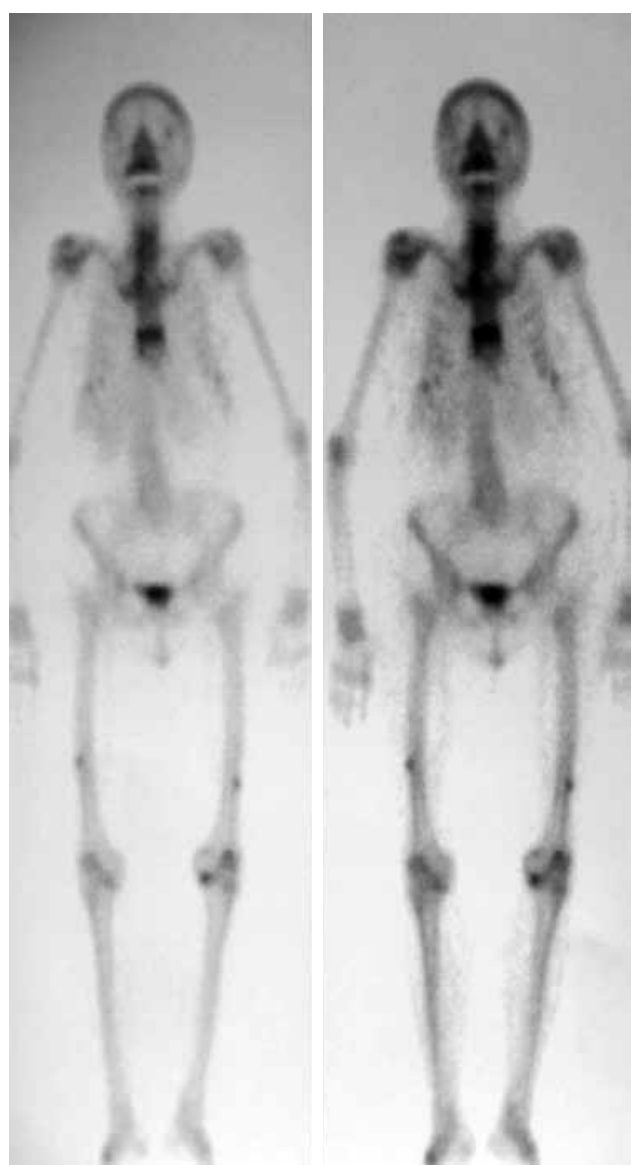

Figure 4. Bone scintigraphy of the second patient.

fractures include prodromal pain, occurring with minimal or no trauma, a thickened diaphyseal cortex and transverse fracture pattern. ${ }^{4}$

Typical femoral periosteal stress reaction was described as cortical thickening in the lateral side of the subtrochanteric region. In addition, Kwek et $a .^{5}$ found bilateral findings of stress reactions or fractures on plain radiographs in 53\% of patients on bisphosphonate therapy. In both of our patients, we observed cortical stress reaction bilaterally.

Radiologically, femoral periosteal stress reaction is described as the presence of the dreaded black line which is a transverse black line traversing the cortex that can be complete or incomplete. ${ }^{6-9}$ Magnetic resonance imaging findings of femoral periosteal stress reaction include focal cortical thickening, bone edema, focal cortical resorption cavities and dreaded black line. There is an association between 
radiographic findings and the presence of clinical symptoms. ${ }^{10}$ Similarly, our patients had thigh pain and difficulty in walking.

Bone scintigraphy of the patients revealed increased uptake in femoral diaphysis. Atypical bisphosphonate associated subtrochanteric and femoral shaft stress fractures show specific appearance on bone scintigraphy characterized by mild uptake in multifocal endosteal thickening of the lateral femoral diaphysis. ${ }^{11}$

Our patients' serum calcium, phosphate, alkaline phosphatase, parathyroid hormone, and 25-hydroxy vitamin D levels were normal. Only urinary deoxyprydinoline levels were elevated. Higher prevalence of hypocalcemia and reduced prevalence of elevated parathyroid hormone levels were reported in patients with atypical femoral fractures. Moreover, lower levels of bone turnover markers were shown at the time of fracture in atypical femoral fractures compared with patients having typical femoral fractures. ${ }^{12}$

We prescribed calcium, vitamin $\mathrm{D}$, and strontium ranelate which also stimulates bone formation. Teriparatide treatment besides calcium and vitamin $\mathrm{D}$ can be used in atypical femoral fractures. ${ }^{13}$ In conclusion, clinically, we should be aware of the cortical stress reaction of femur in patients on long-term bisphosphonate therapy and consider both the risks and the benefits so as to plan treatment.

\section{Declaration of conflicting interests}

The authors declared no conflicts of interest with respect to the authorship and/or publication of this article.

\section{Funding}

The authors received no financial support for the research and/or authorship of this article.

\section{REFERENCES}

1. Mori S. SSBT (severely suppressed bone turnover). Clin Calcium 2013;23:365-70.
2. Jo YR, Kim HW, Moon SH, Ko YJ. A case report of long-term bisphosphonate therapy and atypical stress fracture of bilateral femur. Ann Rehabil Med 2013;37:430-2.

3. Saleh A, HegdeVV, Potty AG, Lane JM. Bisphosphonate therapy and atypical fractures. Orthop Clin North Am 2013;44:137-51.

4. Rizzoli R, Akesson K, Bouxsein M, Kanis JA, Napoli $\mathrm{N}$, Papapoulos $\mathrm{S}$, et al. Subtrochanteric fractures after long-term treatment with bisphosphonates: a European Society on Clinical and Economic Aspects of Osteoporosis and Osteoarthritis, and International Osteoporosis Foundation Working Group Report. Osteoporos Int 2011;22:373-90.

5. Kwek EB, Goh SK, Koh JS, Png MA, Howe TS. An emerging pattern of subtrochanteric stress fractures: a long-term complication of alendronate therapy? Injury 2008;39:224-31.

6. Porrino JA Jr, Kohl CA, Taljanovic M, Rogers LF. Diagnosis of proximal femoral insufficiency fractures in patients receiving bisphosphonate therapy. AJR Am J Roentgenol 2010;194:1061-4.

7. Koh JS, Goh SK, Png MA, Kwek EB, Howe TS. Femoral cortical stress lesions in long-term bisphosphonate therapy: a herald of impending fracture? J Orthop Trauma 2010;24:75-81.

8. Brukner P, Fanton G, Bergman AG, Beaulieu C, Matheson GO. Bilateral stress fractures of the anterior part of the tibial cortex. A case report. J Bone Joint Surg $[\mathrm{Am}]$ 2000;82:213-8.

9. Bergman AG, Fredericson M, Ho C, Matheson GO. Asymptomatic tibial stress reactions: MRI detection and clinical follow-up in distance runners. AJR Am J Roentgenol 2004;183:635-8.

10. Png MA, Koh JS, Goh SK, Fook-Chong S, Howe TS. Bisphosphonate-related femoral periosteal stress reactions: scoring system based on radiographic and MRI findings. AJR Am J Roentgenol 2012;198:869-77.

11. Probst S, Rakheja R, Stern J. Atypical bisphosphonateassociated subtrochanteric and femoral shaft stress fractures: diagnostic features on bone scan. Clin Nucl Med 2013;38:397-9.

12. Franceschetti $P$, Bondanelli M, Caruso G, Ambrosio MR, Lorusso V, Zatelli MC, et al. Risk factors for development of atypical femoral fractures in patients on long-term oral bisphosphonate therapy. Bone 2013;56:426-31.

13. Huang HT, Kang L, Huang PJ, Fu YC, Lin SY, Hsieh $\mathrm{CH}$, et al. Successful teriparatide treatment of atypical fracture after long-term use of alendronate without surgical procedure in a postmenopausal woman: a case report. Menopause 2012;19:1360-3. 